\title{
BLIND ASSISTANCE USING ARTIFICIAL INTELLIGENGE
}

\author{
Aakifah Fathima Shariff, Aisiri B.S, M. Radhika, Sneha S \\ Department of Electronics and Instrumentation Engineering \\ Dayananda Sagar College of Engineering, Bengaluru, Karnataka, India
}

\begin{abstract}
To help the visually impaired and blind people to be independent, this project proposes a portable electronic assistive device based on Artificial Intelligence. This device utilizes different sensors including a GPS sensor, ultrasonic sensor, and a camera module for sensing the surroundings. Raspberry $\mathbf{P i}$, a small computer, processes all the sensor data and provides information of the surroundings through the headphone and thus makes blind people's life easier. This device has a special feature, which provides the location of the blind person to his/her caretaker with the help of a GPS tracker.
\end{abstract}

Keywords - Artificial Intelligence, object detection, text to speech conversion, obstacle and distance measurement, location tracking, blind.

\section{INTRODUCTION}

As per the World Health Organization, there are around 253 million outwardly debilitated individuals, in which 36 million are visually impaired and 217 million experience the ill effects of moderate to extreme vision impedance. Eyesight plays a major role in collecting most of the information from the real world and that information will be processed by the brain, visually impaired people suffer inconveniences in their daily and social life. Blindness or visual impairment is a condition that affects many people around the world. This condition leads to the loss of a valuable sense of vision. Worldwide there are millions of visually impaired people, where many of them are blind. The need for assistive devices was and will be continuous. There is a wide scope of route frameworks and apparatuses existing for outwardly hindered people. The visually impaired individual genuinely requires distinguishing objects. Recently different types of smart canes are being used, however, these have constraints. The cane is unhandy in public places because of the long length and also has a limitation in sensing the surroundings. A Smart Cane comes with its disadvantages. To help the visually impaired and blind people to be independent, this project proposes a portable electronic assistive device based on Artificial Intelligence. In this project, the artificial intelligence-based assistive device is developed which incorporates a small computer i.e a Raspberry pi, camera, GPS module, and an ultrasonic sensor.
This device provides sufficient information about the surroundings and the possible dangers or obstacles, through wearable headphones along with the distance it is located at, and the blind person can also be located using a GPS tracker, thus making visually impaired people non-dependent which can help them to lead a normal life.

\subsection{Objective of the project}

The objective of this project is to come up with a hassle-free solution for the blind, in this case, a wearable device to help them navigate and interact with society.

The proposed technology converts the visual data by image and video processing into an alternate rendering modality that will be appropriate for a blind user. The alternate modalities can be auditory, haptic, or a combination of both. Therefore, the use of artificial intelligence for modality conversion, from the visual modality to another.

\subsection{Scope of the project}

$>$ Use of Raspberry pi v4 will increase the computational speed.

$>$ Probability of accuracy of the object detected right will be more than $70 \%$

$>$ A low cost navigating system is built.

$>$ Saves from serious accidents because of its bigger range detection

$>$ GPS module allows the blind person to be under constant attention.

\section{LITERATURE REVIEW}

A literature search on Visual Impairment identified more than 1000 publications out of which they are shortlisted to a count of 353. This work is carried basing on majorly the Obstacle Detection by Visual Impaired people. Among 353 there are 50 National and International Conferences, 139 National and International Journal's, 40 Thesis produced by Research Scholar's and Master of Degree graduate's from across the world and 100 other publications which are published by graduates, postgraduates and other Research Scholars of standards world's top University's on the Obstacle Detection by Visual Impaired people in between 2004 to 2018 and some other papers from reputed forums. 
2.1 Papers based on the keyword 'Ultrasonic Sensors' Shruti Dambhare, A.Sakhare et al. (2011) [1] proposed "Smart stick for Blind: Obstacle Detection, Artificial vision and Real-time assistance via GPS". In this paper they have given the solution as the replacement for the oldest mobility aid white cane by providing the new system architecture and model explanation of Electronic Travel Aid (ETA). A more safe and secure navigation is provided by the proposed combination of various working units makes a real time system that monitors position of the user and provides dual feedback. And algorithms used for implementing the model are scene segmentation, depth map processing and bounding box estimation. Dada Emmanuel Gbenga, Arhyel Ibrahim Shani, et al. (2017) [2] proposed "Smart Walking Stick for Visually Impaired People Using Ultrasonic Sensors and Arduino". In this paper a modified cane with some electronics components and sensors, the electronic aiding devices is designed to solve such issues. The Ultrasonic sensor is used to detect all kinds of obstacle. When the user comes across a patch of water, water sensor is used to detect if there is water present in front of him. The cane is accomplished to identify the obstacles and humidity in the pathway. Therefore, with the use of ultrasonic sensors, Arduino Uno and other devices that implement audio commands to alert the user of what is in his path of movement a better device can be constructed. O. O. Olakanmi, et al. (2014) [3] proposed "A Multidimensional Walking Aid for Visually Impaired Using Ultrasonic Sensors Network with Voice Guidance", is capable of detecting the direction and position of obstacle. The performance and functionality are also improved by the addition of alert light, and voice guidance signal which is connected to a miniature headset. The voice module guides the user. This multidimensional walking cane was able to detect obstacles within the range of $0 \mathrm{~m}$ to $1 \mathrm{~m}$ at the left, right and front of the stick with an appropriate voice alert. Various traditional methods are available to help the blind individuals for easy movement in both familiar and unfamiliar environments like the use of walking canes, use of guide dogs which provide assistance in navigation or by the help of another human with perfect sight.

\subsection{Wearable device mode of Obstacle Detection}

Parth Dhall, Pankaj Sharma et al. (2016) [4] proposed "A Review Paper on Assistive Shoe \& Cane for Visually Impaired People". In this paper to provide a talkative assistance for a visually impaired person they provided the system architecture and the block diagram of the assistive shoe. In this they implemented the entire work as two modules, one the shoe module, in which a Bluetooth chip, PIC microcontroller along with three IR Sensors are used. Suryavanshi, Mayuri et al. (2016) [5] proposed "Assistance for visually impaired people". In this they have given the system architecture for the shoe which detects the obstacles. This shoe model is implemented by using the Arduino uno board on which all the circuit components is deployed on to. And for the obstacle detection they used the ultrasonic sensors and other hardware components they used are Micro SDHC Card Series and Bluetooth. N. Rama Murthy, N. Sudha, et al. (2016) [6] proposed "Smart Navigation System for Visually Challenged People". In this provided the system architecture of waist belt and shoe through which obstacles are detected by the visual impaired people. In this working is done in such a way that it uses 2 sensors at spectacles, 3 sensors at waist belt which are separated by $12 \mathrm{~cm}$ from each other and 2 sensors in shoe one facing the front and one facing the down.

\subsection{Papers basing on GPS/GSM Technology}

R. Radhika, P.G. Pai, S. Rakshitha and R. Srinath, et al. (2016) [7] proposed "Implementation of smart stick for obstacle detection and navigation". This paper refers to the obstacle detection with the help of ultrasonic sensor and IR sensor connected to Arduino ATMega 328P which has 14 digital input/output pins Ultrasonic sensor detects the range of $3 \mathrm{~m}$ and IR sensor detects small objects at a range of $50 \mathrm{~cm}$, angle $+/-245$ degrees upwards and downwards. Arduino is the microcontroller used. By Ljupko Šimunović, Velimir Anđelić, Ivan Pavlinušić et al. (2012) [8], Alberto Rodríguez, Luis M. Bergasa et al. (2012) [9], in this paper they used the stereo camera for capturing the pictures of the location further which are processed by using the image processing technique namely Dense Disparity Map. In which it processes in such a way that it detects the edges of the captured pictures for easy detection of the obstacles. By using those pictures it is made easy to track the user by equipping the GPS module to the user Somnath koley, Ravi Mishra et al. (2012) [10], S. Madulika, S. Mohan et al. (2013) [11], Vishwa Rupa, B Santosh et al. (2014) [12], these authors introduced the usage of Braille capacitive touch keypad on the controller board. It is very helpful for the blind users because they are unable to see the digit buttons. For that as interface for them they used the Braille language. And they equipped the canes with different sensors like Ultrasonic, LDR, Soil moisture sensor, temperature sensor and the major part GPS module through which the location tracking of the user is done. Due to this facility the caretaker of the blind person can know the live location of the person.

\subsection{Papers basing on Mobile Applications}

Lilit Hakobyan, Jo Lumsden et al. (2013) [13], Nektarios Paisios et al. (2012) [14], Adbel Iiah Nour et al. (2013) [15], in this they discussed the innovative assistive applications designed for the visually impaired by using the current technologies that are either delivered via mainstream devices and can be used while in motion (e.g., 
mobile phones) or embedded within an environment that may be in motion (e.g., public transport) or inside of which the user may be in motion. Ben Leduc-Mills, Halley Profita et al. (2013) [16], Aswathy, Dilraj et al. (2015) [17], proposed a mobile application in which it has two screens namely the main screen and the settings screen. It can be switched from one another just by pressing the provided button. The main screen shows the readings from the 3 ultrasonic sensors in real-time and has a single button for entering the settings screen. A pilot study testing the performance of the ioCane with blind cane users showed a $47.3 \%$ improvement in obstacle avoidance. Karen Soares, et al. (2014) [18], in these thesis papers author proposed a cane for assisting the blind or visual impaired person in shopping activities. And for tracking the location of the user the cane is connected to Wi-Fi through which the location based on the GPS and GSM is transmitted to the person who is care taking and in other thesis author designed the android application basing on SVM and for supporting the SVM they used Open CV android SDK. And they the results of pilot study which given the accuracy approximated to $82.5 \%$.

\section{HARDWARE REQUIREMENTS}

Hardware Components that we will be dealing with:

3.1 RASPBERRY PI V4: It is a low-cost, open-source single board computer used for the complex applications, it is the main hardware controller, integrates all the other components (Camera, IR, Ultarsonic, GPS, Speaker). ML model, TTS, Obstacle/Object detection everything is controlled \& monitored by this. Generally, the raspberry pi is small in size like a credit card designed for the educational purpose. It will improve the programming skills \& hardware programming at the higher education in the schools or colleges. The raspberry pi is much slower than the laptop or desktop and it uses the Linux operating system. It comparatively costs lesser.

3.2 RASPBERRY PI CAMERA MODULE: The device has 5MP resolution camera which is interfaced to raspberry-pi through CSI (Camera Serial Interface). Camera sends video signals to the raspberry-pi and further process will be done by program. The v2 Camera Module has a Sony IMX219 8- megapixel sensor (compared to the 5-megapixel Omni Vision OV5647 sensor of the original camera).

3.3 MEMORY CARD 16 GB: To properly function and run an OS, all Raspberry Pi require a micro SD card and the Raspberry Pi 4 is no exception. Raspberry Pi series of boards have no integrated eMMC/onboard or other non-volatile storage, therefore there is no way to boot the Raspbian OS without an SD card or external hardware.

3.4 POWER ADAPTER: The power supply requirements differ by Raspberry Pi models. All models require a 5.14 supply, but the current supplied generally increases according to the model.

3.5 GPS MODULE: GPS stands for Global Positioning System which provides location information to the GPS receiver anywhere in the Globe. It is a universal radio navigation system established by the U.S Department Of Defence (DOD) to give information about the location for military applications. It is used for location tracking, interfaced through UART/USB and output is in NEMA format.

3.6 ULTRASONIC SENSOR: It is used for measuring distance of object in "cm", its range is about $3 \mathrm{~cm}$ to $300 \mathrm{~cm}$. Interfaced through GPIO and output is in terms of pulse width. The ultrasonic sensor deals with the guideline of SONAR and RADAR framework which is utilized to decide the separation to an item. A ultrasonic sensor produces the high-recurrence sound (ultrasound) waves. The blind assistance is integrated with the ultrasonic sensor. Our proposed venture first uses ultrasonic sensors to identify obstructions ahead utilizing ultrasonic waves. Distance is measured by sending out a sound wave at a particular frequency and waiting for that sound wave to bounce back. It works by sending out a burst of ultrasound and waiting for the echo when it bounces off of an object. It pings the obstacles coming in the way with ultrasound.

3.7 SPEAKER (EARPHONES): What an earphone does is, it converts electrical signals into sound waves. It is a general-purpose mini speaker used for the playback of detected object, distance and warning messages.

3.8 BOOST CONVERTER (3.7V TO 5V): A boost converter is one of the simplest types of switch mode converter that exist. As the name itself claims, it takes an input voltage and boosts or increases it. All it consists of is an inductor, a semiconductor, a diode and a capacitor. MT3608 boost converter used to supply power to Raspberry-Pi. Battery is $3.7 \mathrm{~V}$ but the Raspberry Pi requires $5 \mathrm{~V}$, for this boost converter is used.

3.9 LITHIUM ION BATTERY (3.7V TO 3000MAH): A lithium-ion battery or Li-ion battery is a type of rechargeable battery used in many applications. Lithium-ion batteries are commonly used for portable 


\section{International Journal of Engineering Applied Sciences and Technology, 2020 \\ Vol. 5, Issue 3, ISSN No. 2455-2143, Pages 396-401 \\ Published Online July 2020 in IJEAST (http://www.ijeast.com)}

electronic goods. For powering the device $6 \mathrm{v}$ battery is required, the average power consumption of the total device is around $1 \mathrm{Amps}$. If $3.2 \mathrm{AH}$ battery is used then the device runs for 3 hours continuously.

\section{SOFTWARE REQUIREMENTS}

The software that we will be dealing with in this project are:

\subsection{JUPYTER NOTEBOOK: DEVELOPMENT} ENVIRONMENT: The Jupyter Notebook is a sort of open-source web application that permits you to make and offer records that contain live code, conditions, perceptions and story content and other such applications.

4.2 PYTHON: PROGRAMMING LANGUAGE: It is a high-level programming language widely used for the Machine-Learning and also has a lot of libraries \& support. Hence it is selected.

4.3 TENSOR FLOW: MACHINE LEARNING NETWORK: It is an open-source, most widely used framework for ML, using this python library the object detection model is designed \& trained. It plays the vital role in the device.

\section{METHODOLOGY}

The proposed device uses Computer Vision and Deep Learning technologies that come under artificial intelligence. This artificial intelligence technology is used for object detection using the camera. Ultrasonic sensors are used for distance measurement. GPS provides location tracking capabilities that enable the care-taker to track blind people.

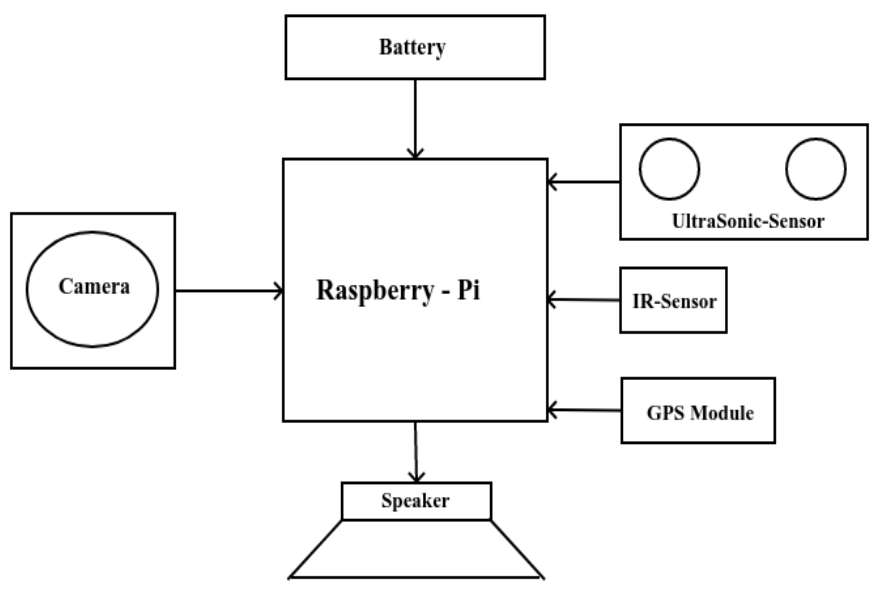

Fig.1. Block Diagram
Fig. 1 shows the block diagram of the device that we have proposed. Raspberry-Pi forms the central part integrating all the components like- sensors, cameras, GPS, and speaker/headphones. Python programming language is used to program the whole system/device which helps raspberry-pi to communicate with all the sensors. Then it processes the sensor data and converts to the human-readable information and finally delivers to the blind people through speakers.

The system consists of different modules, which are as follows-

5.1 Object Detection: First the ML Model has to be created \& trained. Once the model is ready, it takes video streams from the camera then the ML model predicts objects using features from the video.

5.2 Text-to-Speech Conversion: The predicted object is in the "ASCII-Text" format, this should be converted to "audio" by using TTS (Text-To-Speech) synthesis and finally playing this audio through the speaker.

5.3 Obstacle \& Distance Measurement: To detect any obstacle in the forward direction of the device, Ultrasonic sensor is used. It will alert the user (Blind Person) by audio-warning through the speaker. The device also employs a distance measurement of the obstacle/object in the sensor's view, the Ultrasonic sensor is used for this purpose. Once the distance is measured it is converted to audio and played to the speaker.

5.4 Location Tracking: To have a track of the Blind the device employs GPS and the current location of the Blind can be shared and tracked by the care-taker.

\section{CODING}

Python programming language is used to program the whole system, following is the flowchart of the whole system. 
International Journal of Engineering Applied Sciences and Technology, 2020

Vol. 5, Issue 3, ISSN No. 2455-2143, Pages 396-401

Published Online July 2020 in IJEAST (http://www.ijeast.com)

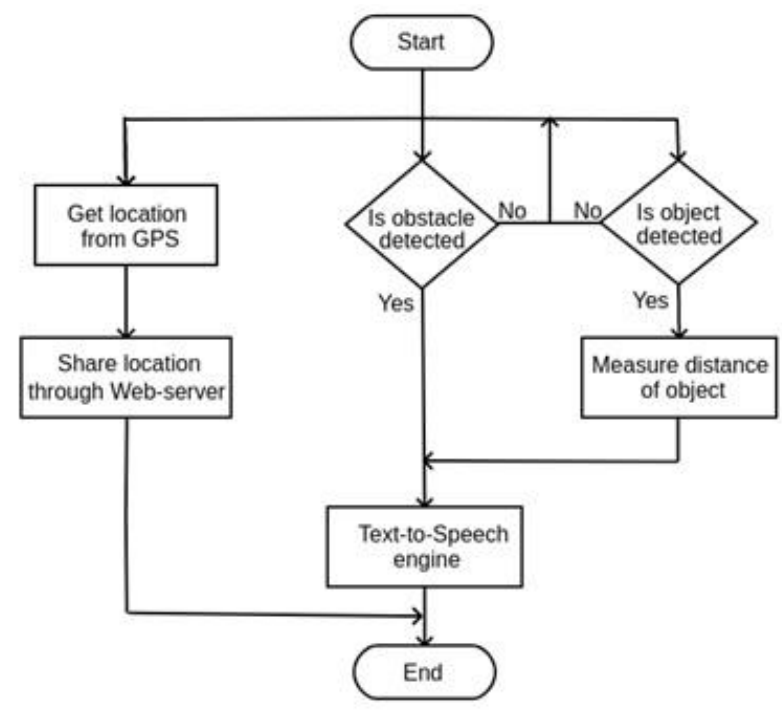

Fig.2. System Flowchart

System consists of three modules, location tracking, obstacle detection and object detection. These three modules runs concurrently.

6.1 Location Tracking: Gets the current position of the device (worn by blind people) from the GPS module, manipulates the data, and fetches the location coordinates (latitude and longitude).

6.2 Obstacle Detection: While the blind person moves around with wearing this device if the blind person is about to hit any obstacle the device's obstacle detection module will alert the person with an audio message through the TTS engine.

6.3 Object Detection: This module continuously detects the object from the camera images, if any object is detected then the distance of the object is measured through an ultrasonic sensor, and finally, the detected object name and the distance are conveyed to the blind by audio message.

\section{RESULT}

The camera captures an image of the object which is detected by the sensor which is further converted into an audio output using Raspberry Pi. We measure the distance of the object using the ultrasonic sensor. Both outputs are heard in an audio. The GPS system helps in locating the exact position of the visually impaired which helps them to navigate to the desired destination.

\section{FUTURE SCOPE}

With the help of this system the visually impaired can move independently and safely. Movement in public places will be much easier now. The user can easily navigate with the help of directions that the system audio provides. The system can be designed with low cost, low power consumption and less cost. It is a much a dynamic option as it requires less space. It is much more efficient compared to systems that are used at present. By providing much simpler wearable devices such as necklace or goggles we can aim to get closer to the future scope of the project. By embedding ultrasonic sensors below the abdomen level it will be helpful for the user to navigate more effectively. Therefore, this system will help in providing a social service to the visually impaired by lowering their day to day challenges.

\section{CONCLUSION}

Using raspberry pi we have implemented a technique that converts text to speech. The hardware outputs have been tested using different samples and we successfully verified the simulation results. The algorithm that is being used here successfully processes the image and outputs the audio clearly. The device has been tested in various environments with various objects and the output was verified successfully. From the survey conducted to our knowledge it seems usage of algorithms from advanced technologies like Machine Leaning, Deep Learning and Artificial Intelligence from all of the proposed approaches will provide the better navigation aid for the Visually Impaired people. The device is compact and has great economic value. It is a very efficient device and is a very helpful solution for the society.

\section{REFERENCE}

[1] Shruti Dambhare, Prof. A. Sakhare, (2011) "Smart stick for Blind: Obstacle Detection, Artificial vision and Real-time assistance via GPS", 2nd National Conference on Information and Communication Technology (NCICT) Proceedings published in International Journal of Computer Applications ${ }^{\circledR}$ (IJCA). (pp 31-33)

[2] Dada Emmanuel Gbenga, Arhyel Ibrahim Shani, Adebimpe Lateef Adekunle, (2017) "Smart Walking Stick for Visually Impaired People Using Ultrasonic Sensors and Arduino." International Journal of Engineering and Technology (IJET), Vol 9, No 5, DOI: $10.21817 /$ ijet/2017/v9i5/170905302

[3] O. O. Olakanmi, (2014) "A Multidimensional Walking Aid for Visually Impaired Using Ultrasonic Sensors Network with Voice Guidance", International Journal of Intelligent Systems and Applications (IJISA), vol. 6, number 8, pp. 53-59, 2014. DOI: $10.5815 /$ ijisa.2014.08.06.

[4] Parth Dhall, Pankaj Sharma, (2015) “A Review Paper On Assistive Shoe \& Cane for Visually Impaired People", International Journal of Scientific Research and Management Studies (IJSRMS) ISSN: 23493771 Volume 3 Issue 2, (pp- 113-117). 
[5] N. Suryavanshi, S. Mayuri, (2016) "Assistance for visually impaired people", International Journal for Research in Applied Science \& Engineering Technology (IJRASET), (pp- 371-375) Volume 4 Issue IV, April 2016, ISSN: 2321-9653.

[6] N. Rama Murthy, N. Sudha,(2016) "Smart Navigation System For Visually Challenged People", International Journal of Industrial Electronics and Electrical Engineering, (pp-45-48) ISSN: 2347-6982 Special Issue, Sep.-2016.

[7] R. Radhika, P.G. Pai, S. Rakshitha and R. Srinath, (2016) "Implementation of Smart Stick for Obstacle Detection and Navigation." International Journal of Latest Research in Engineering and Technology, vol. 2, number 5, (pp. 45-50).

[8] Ljupko Šimunović, Velimir Anđelić, Ivan Pavlinušić, (2012) "Blind people guidance system", Central Conference on Information and Intelligent Systems, Faculty of Organization and Informatics, Sep 19-21, (pp 1-8)

[9] Alberto Rodríguez, Luis M. Bergasa (2012), "Obstacle Avoidance System for Assisting Visually Impaired People", Proceedings of the 2012 IEEE Intelligent Vehicles Symposium Workshops, ISBN: 978-84-695-3472-4,DOI:

10.1109/ICARCV.2014.706461

[10] Somnath koley, Ravi Mishra, (2012) "Voice Operated Outdoor Navigation System For Visually Impaired Persons", International Journal of Engineering Trends and TechnologyVolume3Issue2- 2012. (pp. 153-157)

[11] S. Madulika, S. Mohan, (2013) "Arm7 Based Electronic Travel Aid System for Blind People Navigation and Monitoring", International Journal of Research in Computer and Communication Technology, Vol 2, Issue 12, December- 2013, (pp.153-155)

[12] Vishwa Rupa, B Santosh, (2014) "Rescue System for Visually Impaired Blind Persons", International Journal of Engineering Trends and Technology (IJETT) - ISSN: 2231-5381 Volume 16 Number 4 Oct 2014. (pp. 153-155)

[13] Lilit Hakobyan, Jo Lumsden, (2013) "Mobile Assistive Technologies for the Visually Impaired", City, University of London Institutional Repository. DOI: 10.1016/j.survophthal.2012.10.004

[14] Nektarios Paisios,(2012) "Mobile Accessibility Tools for the Visually Impaired", Courant Institute of Mathematical Sciences New York University, PHD Thesis 2012, Available from URL: https://cs.nyu.edu/media/publications/nektariosp.pdf

[15] Adbel Iiah Nour, (2013) "Automated Mobility and Orientation system for Blind or Partially Sighted People", International Journal on Smart Sensing and
Intelligent Systems, vol. 6 no. 2, April 2013, DOI: 10.21307/ijssis-2017-555

[16] Ben Leduc-Mills, Halley Profita, (2013), "ioCane: A Smart-Phone and Sensor-Augmented Mobility Aid for the Blind", Submitted as a Computer Science Technical Report to the University of Colorado, Boulder libraries on September 23, 2013. URL: file:///C:/Users/aakifah/Downloads/iocaneASmartPho neAndSensorAugmentedMobilityAidForTheB.pdf

[17] Aswathy, Dilraj, (2015) "Rf Based Talking Signage For Blind Navigation", International Journal on Cybernetics \& Informatics (IJCI) Vol. 4, No. 2, April 2015, DOI: $10.5121 /$ ijci.2015.4217

[18] Karen Soares, (2014) "SmartGuia: Shopping Assistant for Blind People", a thesis presented to Department of de Engenharia Informática, Universidade de Coimbra, URL: https://core.ac.uk/download/pdf/43574861.pdf 